\title{
On a new process for cusp irregularity production
}

\author{
H. C. Carlson ${ }^{1,2}$, K. Oksavik ${ }^{3}$, and J. Moen ${ }^{1}$ \\ ${ }^{1}$ Department of Physics, University of Oslo, Oslo, Norway \\ ${ }^{2}$ Utah State University, CASS, Logan, UT, USA \\ ${ }^{3}$ Arctic Geophysics, The University Centre in Svalbard, Longyearbyen, Norway
}

Received: 23 January 2008 - Revised: 10 June 2008 - Accepted: 19 August 2008 - Published: 23 September 2008

\begin{abstract}
Two plasma instability mechanisms were thought until 2007 to dominate the formation of plasma irregularities in the F region high latitude and polar ionosphere; the gradient-drift driven instability, and the velocity-shear driven instability. The former mechanism was accepted as accounting for plasma structuring in polar cap patches, the latter for plasma structuring in polar cap sun aligned arcs. Recent work has established the need to replace this view of the past two decades with a new patch plasma structuring process (not a new mechanism), whereby shear-driven instabilities first rapidly structure the entering plasma, after which gradient drift instabilities build on these large "seed" irregularities. Correct modeling of cusp and early polar cap patch structuring will not be accomplished without allowing for this compound process. This compound process explains several previously unexplained characteristics of cusp and early polar cap patch irregularities. Here we introduce additional data, coincident in time and space, to extend that work to smaller irregularity scale sizes and relate it to the structured cusp current system.
\end{abstract}

Keywords. Ionosphere (Ionosphere-magnetosphere interactions; Polar ionosphere) - Magnetospheric physics (Auroral phenomena)

\section{Introduction}

The plasma in the polar cap ionosphere is highly structured, and the nature of this structure and the phenomena leading to its structuring has been the focus of much research for the past few decades (Basu and Valladares, 1999). The structuring is of scientific interest, as a process fundamental to understanding the Earth's near space environment and space plasmas more generally, and of practical consequence to re-

Correspondence to: H. C. Carlson

(herb.carlson@london.af.mil) ception of satellite signals for communication and GPS navigation. For the past twenty years this has led to active studies of what has come to be known as polar cap patches (Crowley, 1996) and polar cap arcs (Valladares and Carlson, 1991). The most intense structuring and scintillation occurs in polar cap patches. Their structuring has also been a subject of intensive study (Basu and Valladares, 1999). Our primary focus in this work is to discuss the processes that structure the plasma in polar cap patches.

The large auroral and polar cap electric fields, currents, and plasma drifts relative to the neutral atmospheric velocity can and do lead to a variety of instabilities. These plasma instabilities cause otherwise relatively smooth plasma to develop inhomogeneous magnetic field-aligned structures over scale sizes of $\sim 10 \mathrm{~s} \mathrm{~km}$ to meters (Basu et al., 1990). Radio waves propagating through these irregularities experience strong scintillation, both amplitude fading and phase fluctuations, up to Giga-Hertz (GHz) frequencies (Basu et al., 1988). These disrupt VHF (30-300 MHz), UHF and GPS navigations systems at L-band frequencies (note scintillation may be observed up to $6 \mathrm{~dB}$ at $3 \mathrm{GHz}$ at equatorial latitudes because the plasma densities there are relatively higher than those commonly found at high latitudes). These strong signal amplitude fades are significant to physics and engineering, including societal and economic impact. At longer wavelengths these same plasma irregularity regions also backscatter radio waves, especially at $\mathrm{HF}(3-30 \mathrm{MHz})$ and VHF wavelengths. This scatter or clutter can blind radar tracking, disrupt or improve HF communications, and serve as an important geophysical diagnostic tool. These structures and the plasma instability processes producing them are a subject of intense research to understand the associated plasma physics and the major effects on satellite radio communications, navigation, radar imaging, and earth-bound HF communication systems.

For two decades two fundamentally different mechanisms have been thought to dominate the processes by which

Published by Copernicus Publications on behalf of the European Geosciences Union. 
POLAR CAP F-REGION STRUCTURES
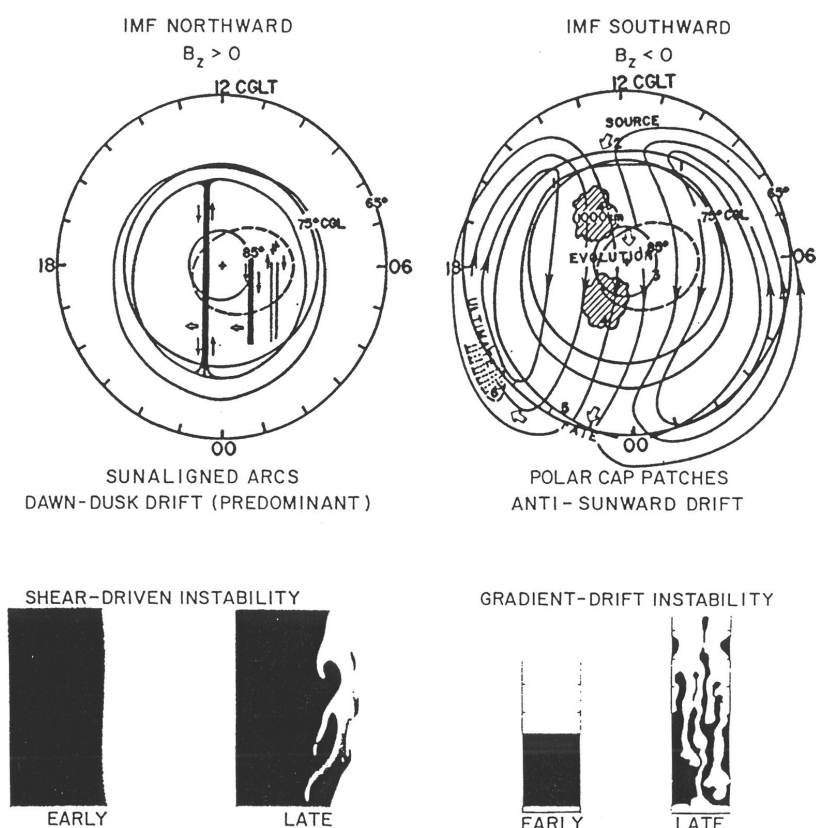

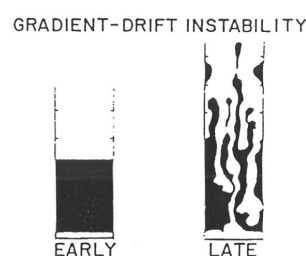

Fig. 1. Conditions characterizing the northward (top left) and southward (top right) IMF state of the polar cap ionosphere, after an extended period dominated by respectively sun aligned arcs and polar cap patches (from Carlson, 1994). The process believed to dominate plasma structuring in sun aligned arcs is the shear driven instability illustrated in the lower left (Keskinen et al., 1988), while the process believed until 2007 to dominate patch structuring is illustrated in the lower right (Keskinen and Ossakow, 1983). The composite figure is adopted from Carlson et al. (2007).

plasma irregularities producing high latitude and polar scintillation are generated; 1) the gradient drift, and 2) the velocity shear driven instability.

1. The gradient-drift (GD) plasma instability mechanism is known to occur for plasma drift, of the correct sign, across a steep plasma-density gradient perpendicular to the Earth's magnetic field at high latitudes (Keskinen and Ossakow, 1983). (Drift in the opposite direction across the plasma density gradient sets up polarization fields that stabilize the plasma against any irregularity formation.) The theory has also been refined to include the effects of high-latitude auroral currents on its quantitative thresholds and behavior. Until 2007 this was believed to dominate structuring in southward IMF polar cap patches.

2. Velocity shear (Kelvin-Helmholtz, KH) driven plasma instabilities were experimentally determined to occur for sufficiently severe plasma velocity shears (Basu et al., 1988, 1990). Keskinen et al. (1988) have developed a Kelvin-Helmholtz instability theory to describe these latter instabilities, including the addition of the re-

finement of ionospheric-magnetospheric electrical coupling. This instability is believed to dominate the structuring of the northward IMF polar cap, in particular polar cap arcs.

To observationally distinguish between these two different instability mechanisms, it is important that for a given percent electron density fluctuation, the plasma velocity fluctuation is severely dependent on whether the mechanism is the velocity-shear instability or the gradient drift instability. For the conditions published in Basu et al. (1988), the fluctuation in local plasma velocity or electric field, for a given percent fluctuation in local electron density $(\Delta E[\mathrm{mV} / \mathrm{m}] \mathrm{vs}$. $\Delta N / N[\%])$, was $\sim 10$ times stronger for the velocity-shear mechanism than for the gradient drift instability. This overall context is summarized in Fig. 1. We shall now examine how this understanding of the past two decades has come to require a revision, as a result of recent discoveries that relate to processes in the cusp and the launching of patches into the polar cap.

\section{The origin of polar cap patches}

The highest plasma density in the polar cap, and the strongest consequent scintillation, is that associated with polar cap patches. Both appear most dramatically in winter when the background polar cap plasma has low densities characteristic of mid-latitude nighttime values, surrounding islands of high densities characteristic of daytime values. Inside these islands, called polar cap patches, the plasma density can be ten times the surrounding density (going from $\sim 10^{11}$ to $10^{12} \mathrm{~m}^{-3}$ ) with associated scintillation going from negligible to saturation. Explaining the source of the plasma leading to these order of magnitude "night to day" plasma density increases was not particularly challenging, they were attributed to daytime sunlit plasma being transported into the polar cap by the large-scale convection patterns (e.g. Heelis et al., 1984).

What has been challenging has been to explain why these high density plasma intrusions, transported across the polar cap from the dayside to the nightside, were found not as a continuous tongue of ionization but in the form of finite islands or patches $\sim 100-1000 \mathrm{~km}$ across. Several mechanisms have been proposed, most of which likely can and do make some patches. Proposed mechanisms include: discrete changes in IMF $B_{y}, B_{z}$, and solar wind speed/density/pressure (e.g. Anderson et al., 1988); plasma flow jets in which "temperature dependent" recombination rates cut continuous tongues of plasma into segments (Valladares et al., 1996, 1999); plasma production by cusp particle precipitation (Walker et al., 1999); transient magnetopause reconnection (Lockwood and Carlson, 1992); and formation of patches on closed field lines well equatorward of the cusp auroral activity (Moen et al., 2006). A better 
question could then be which mechanism makes the most patches.

The Lockwood and Carlson (1992) mechanism was based on transient reconnection at the magnetopause (Cowley and Lockwood, 1992). By this mechanism a burst of magnetopause reconnection applies a voltage (in its own rest frame) along the X-line in the low-latitude magnetopause, which projects down to the ionosphere where (absent of significant field-aligned voltage drop) it applies to the merging gap in its own rest frame. If the merging gap is fixed in the Earth frame one gets a continuous tongue of ionization. However, if the merging gap moves equatorward at the onset of reconnection it crosses plasma whose flow into the polar cap accelerates with time. At turnoff, when reconnection stops and there is no electric field along the merging gap, the plasma flows poleward with the merging gap boundary, and the open-closed boundary relaxes back towards an equilibrium configuration (Cowley and Lockwood, 1992). After the event adjacent flux tubes can have arrived from very different source locations, and patches can occur if the source reservoirs are of very different densities (e.g. across the solar terminator).

This mechanism can be tested by looking for a specific sequence of signatures; an optical flash from particle precipitation upon a flux tube opening, extra electron gas heating by particles, ion temperature rise associated with enhanced ion velocity (dissipation of Pointing flux in Joule heating), ion velocity spurt, and a high density plasma boundary entering the polar cap. These five signatures $(\mathrm{Ne}, \mathrm{Vi}, \mathrm{Ti}, \mathrm{Te}$, and optical emission flash), with the predicted relative timing and locations, were observed and reported by Carlson et al. (2004) as strong evidence of repeated signatures of magnetic reconnection in the cusp during southward IMF conditions over the EISCAT Svalbard Radar (ESR) during January 2001. Preceded by an optical flash (precipitating particles move most quickly) the four plasma parameter boundaries moved poleward in unison, each with the predicted respective relative delay (heating time constant and onset of motion).

The following winter, December 2001, an experiment was designed and performed capable of mapping the high-density plasma in polar cap patches during their transit between their lower-latitude plasma reservoir and their ultimate trajectory within the polar cap. These data included simultaneous observations of north-south meridian scans with the ESR, the Troms $\varnothing$ EISCAT VHF and UHF radars looking north at low elevation near the ESR field-of-view, and optical data from all-sky imaging photometers (ASIPs) and meridian scanning photometers (MSPs). Carlson et al. (2006) reported a sequence of five successive patches and mapped them into the polar cap during a $40 \mathrm{~min}$ interval near local magnetic noon, reproduced here in Fig. 2. Each event showed the motion of distinct features in the measured plasma parameters, but in addition MSP and ASIP data showed that the optical boundary moved in synchronization with the sharp boundaries of enhanced Ne, Te, Ti, and Vi. This incidentally di- rectly confirmed one class of strongly enhanced emissions $(630.0,557.7$, and $777.4 \mathrm{~nm})$ of poleward moving auroral forms (PMAFs) to be optical markers of reconnection events. It then linked a large body of one class of PMAF studies to become supporting information about ionospheric signatures of magnetic reconnection events at the magnetopause.

This work provided direct observations of injection events of subauroral plasma into the polar cap, establishing reconnection events at the magnetopause as a dominant mechanism for the creation of patches during southward IMF conditions. Thus this dominant creation source of polar cap patches is intrinsically associated with bursts of plasma flow shears. Transient reconnection at the magnetopause drives a transient electric field that entrains lower latitude plasma into a transient poleward flow channel and transports the plasma parcel across the prior cusp location and into the polar cap.

\section{Patches and flow shears}

If flow shears are intrinsic to the mechanism producing polar cap patches, this immediately raises the question of what role does the shear driven instability play in the structuring of patches. For two decades the community has viewed the gradient drift instability as the plasma instability mechanism that structures plasma in polar cap patches, but the new knowledge that patches experience strong flow shears in their creation process puts it in a new context. This realization led Carlson et al. (2007) to explicitly address this question anew.

For the data in Fig. 2, which relates to patches driven by magnetopause reconnection, we can rule out two alternate patch generation mechanisms. First, we can rule out segmentation of a continuous tongue of ionization through the cusp by enhanced recombination in a high velocity plasma jet. We observe increased plasma density in high velocity streams away from the cusp (carrying high density plasma into the polar cap), but we do not see decreased density in the high speed streams. Plasma flows $\gg 1 \mathrm{~km} / \mathrm{s}$ should erode plasma at lower altitudes (much below $250 \mathrm{~km}$ ) and should play a role for patches from the sunlit lower altitude cusp such as at Sondrestrom (Valladares et al., 1996). However, in our case the plasma is several neutral molecular scale heights higher in altitude. Secondly, if the plasma in the plasma jet is at sufficiently low altitude that the ions have large neutral molecular densities with which to react, enhanced recombination must occur, and for such conditions patches could become segmented. However, here too the patch formation process would be at a strong velocity shear. In fact most reported observations of patch generation regions also report associated flow jets. Thus velocity shears apply to an even broader set of patches than the dominant class reported here.

In Fig. $2 \mathrm{a}$ and $\mathrm{m}$ we see that the poleward edge of the subauroral F-region plasma reservoir, from which patches can be drawn, is near $72.5^{\circ}$ magnetic latitude, as mapped by both the ESR and the EISCAT VHF Beam 1. Note that in the EISCAT 

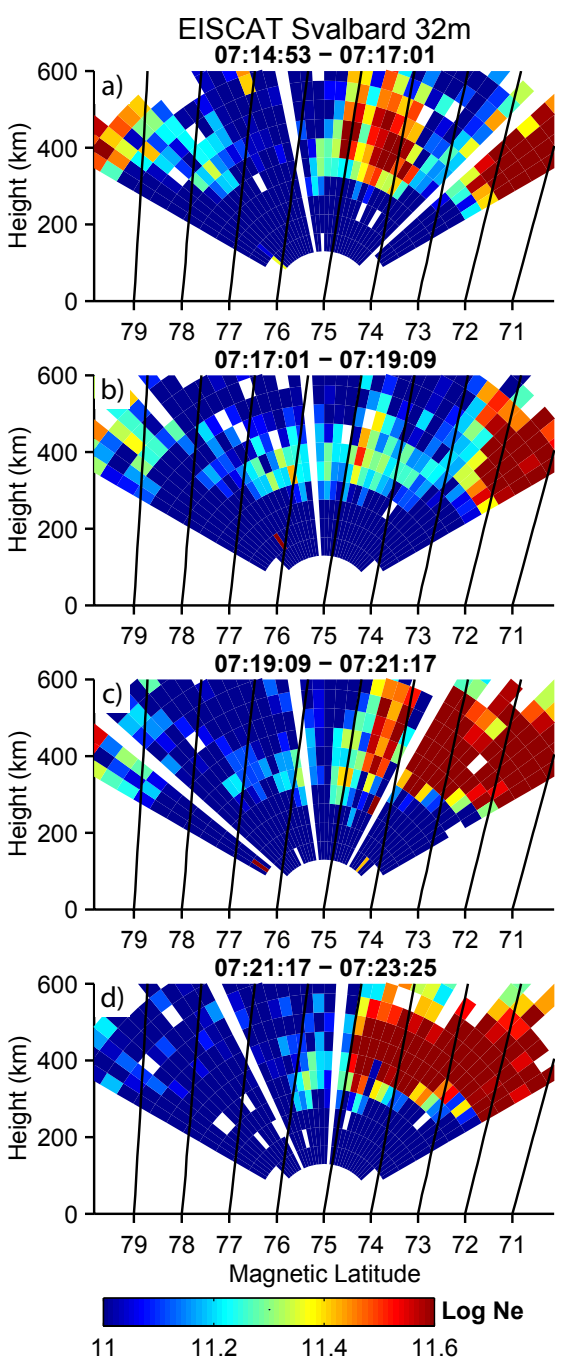
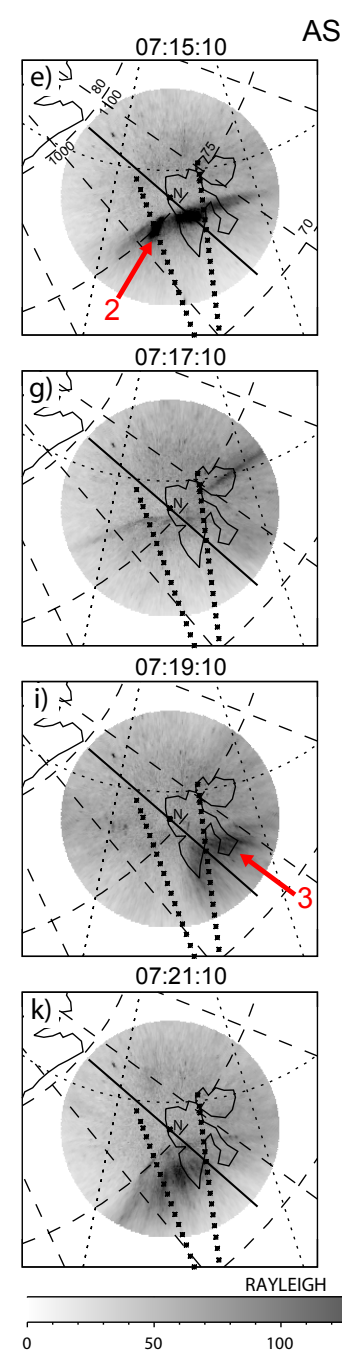

ASIP
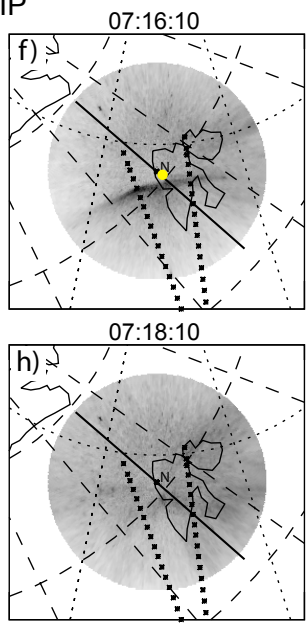

$07: 20: 10$

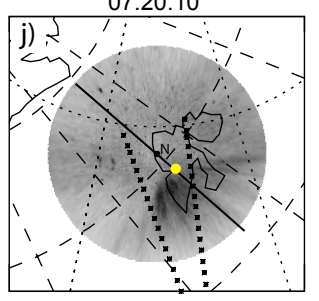

$07: 22: 10$

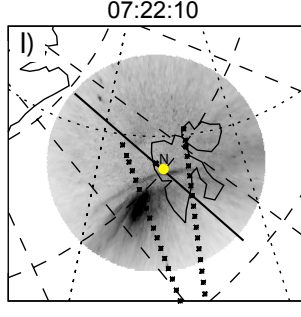

$777.4 \mathrm{~nm}$
EISCAT VHF Beam 1

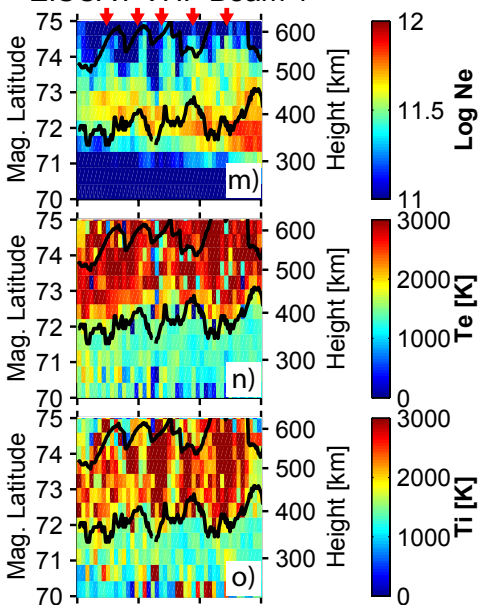

750000
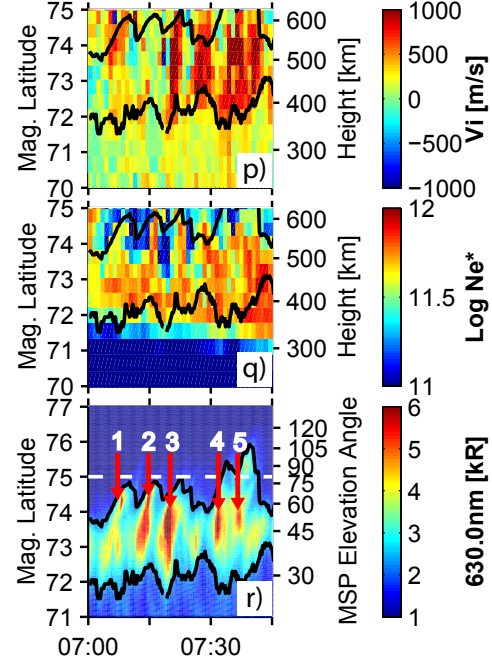

Universal Time

Fig. 2. The upper left panel (a) shows ESR measurements of the cusp plasma boundary just above $72^{\circ}$ and the previously injected patch farther to the north. The next three panels (b-d) show the injection of the next patch. The central series of ASIP images (panels e-l) show optical emissions marking the associated particle precipitation boundary that is surging poleward with the reconnection event. Both the electron density measurements $(\log \mathrm{Ne})$ and the ASIP images show the speed with which the event boundaries move. The panels on the right show plasma properties from the EISCAT VHF radar that was looking over Svalbard (m-q) and meridian scanning photometer data from Ny-Ålesund (r). These data show five successive patches that are created and injected across the cusp and into the polar cap. Further details are given in Carlson et al. (2006), which is the source of this figure.

VHF beam the altitude depends on range, due to Earth's curvature, as indicated by the right hand scales in Fig. $2 \mathrm{~m}-\mathrm{q}$. This means that the F-region altitude ESR profiles in Fig. 2a$\mathrm{d}$ will appear as a slice in range where the radar line-of-sight cuts across the F layer. The ASIP data in Fig. 2e-1 show the optical footprint of newly reconnected magnetic field lines projected down to the ionosphere/thermosphere. Note how rapidly the optical and plasma boundaries move poleward.

The data set in Fig. 2 had several complimentary instruments operating and collecting data. One such instrument was a receiver collecting signals from a radio beacon on a satellite. The five successive patches seen breaking into the polar cap in Fig. 2, passed between the line-of-sight of a pair of ground receivers; one at Longyearbyen (near the ESR), and another near Ny-Ålesund (about $100 \mathrm{~km}$ north). Any strongly structured plasma passing through the line-ofsight from the satellite to the ground receiver would produce a scintillation signature. Figure 3 shows the results of this experiment. As reported by Carlson et al. (2007), each patch registered a strong scintillation signature approaching saturation by the time is passed over Ny-Ålesund. The red curve corresponds to the right hand scale in the top panel of Fig. 3, where the scintillation saturated at 1.0. In the figure the $\mathrm{Ny}-$ Ålesund measurements are shifted by two minutes to account 
for the measured plasma drift velocity over Longyearbyen ( $75^{\circ}$ magnetic latitude).

If the plasma velocity shear is important to rapidly initiate plasma irregularity structures, we should see a scintillation signature for each of the five patches that are launched into the polar cap over Svalbard (from equatorward to poleward). The five red arrows in Fig. 3 mark the passing time at the ESR latitude through the VHF beam (dashed line on ASIP images in Fig. 2e-1). Carlson et al. (2007) estimated the growth rates for the gradient drift and for the shear driven instability for the conditions of these patches, and they showed that the gradient drift is too slow to produce such scintillation amplitudes, while estimates of the shear driven instability can explain the observed amplitude quite well. It is not the slowly growing gradient drift instability that drives the patch structuring, as believed for the past two decades, but the rapid onset of the shear driven instability that drives the initial patch structuring. The gradient drift instability alone would start operating on weak plasma irregularities and take a large number of growth rate time constants to reach strong structuring. The rapid rise time of shear driven (or KelvinHelmholtz, KH) instability quickly produces initial structuring. After the patch plasma becomes structured in passing through the cusp, it "seeds" the plasma with large amplitude structures, and the gradient drift (GD) then operating on strongly pre-structured plasma is enabled to contribute in only a few growth times. This means a new two-step process must be adopted. No new mechanism is required, but a new process must be applied to a pair of previously known instability mechanisms (GD and $\mathrm{KH}$ ).

In summary, after developing in 2001 an observing mode for the ESR (Carlson et al., 2002) that was capable for the first time of testing the Lockwood and Carlson (1992) mechanism, Carlson et al. (2004, 2006) established that this magnetic reconnection based mechanism, is a dominant mechanism for creating polar cap patches. The same data body showed associated strong flow shears to be commonly observed in the cusp. This immediately raised the question of how important these shears might be for creating plasma irregularities in the entering patch plasma, due to the sheardriven instability. Carlson et al. (2007) showed that this shear driven instability dominates initial rapid formation of irregularities in polar cap patches, in contrast to the gradient drift instability that was previously thought to dominate. The GD instability presumably becomes dominant once the patch has entered and becomes part of the general background largescale transpolar convection and shear forcing relaxes. The conclusion was that correct modeling of cusp and early polar cap patch structuring will not be accomplished without allowing for this compound process.

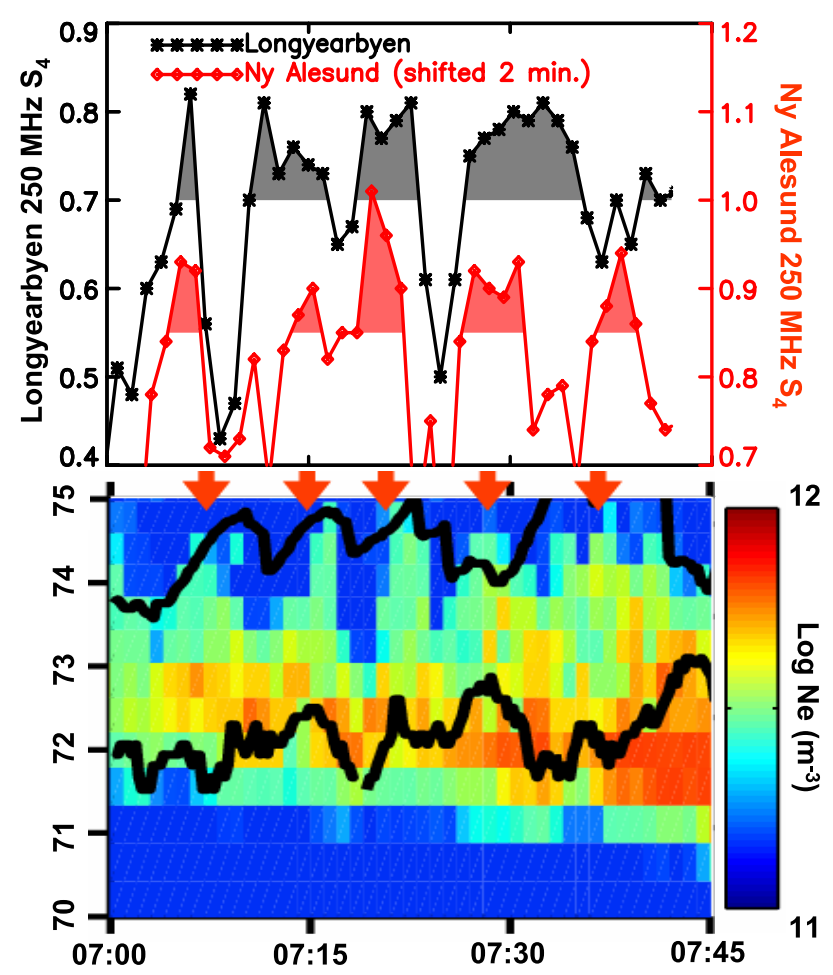

Fig. 3. The lower panel shows a sequence of five newly created patches entering the cusp, captured in polar flow from initially subauroral return flow. The red arrows show previously published passing times over Longyearbyen of newly formed patches (Fig. 1m). The left axis is magnetic latitude, and the color scale shows the electron density ( $\log \mathrm{Ne}$ ) along the line-of-sight of the EISCAT VHF radar. Due to Earth's curvature, $71^{\circ} / 73^{\circ} / 75^{\circ}$ magnetic latitude is at $300 / 450 / 620 \mathrm{~km}$ altitude. The upper panel shows the passage of five strong scintillation events over Longyearbyen (dark grey) coincident with the entering patches (red arrows). The red areas show more severe scintillation when the same patches have moved $\sim 100 \mathrm{~km}$ poleward to $\mathrm{Ny}$-Ålesund. The time scale of the red curve is shifted $\sim 2$ min to account for the flow speed. The S4 scintillation index has grown from less than 0.8 over Longyearbyen to greater than 0.9 over $\mathrm{Ny}$-Ålesund.

\section{Flow shears and instabilities}

The new process is built by combining two established mechanisms which act sequentially and make the cusp plasma structure more rapidly and more severely. By pre-initializing polar cap patches on entry into the polar cap, they structure more fully within the whole patch, instead of slowly working forward from the trailing edge as observed in and across the polar cap (Heelis, 1984). Initially, strong shears are associated with entry of patches through the cusp into the polar cap. These shears (by the shear driven or KH instability) create strong structure much faster than gradient-drift alone, enabling a rapid turn on of irregularities in the cusp inflow region. These irregularities also "seed" structures large enough 
to accelerate the subsequent GD instability process in rapidly building stronger irregularity structure deeper in the polar cap. The KH and GD instabilities act along different (quasiorthogonal) orientations, leading to a more rapid 2-D filling of irregularities in patches than could be explained before the process proposed herein. This in-tandem operation of the velocity shear instability followed by the gradient drift instability, in the framework of linear instability theory, should then structure the plasma patches in both the anti-sunward and dawn-dusk directions.

Very important is that the plasma flow includes transients driven by the magnetic reconnection mechanism. If plasma entered the polar cap through the cusp only with a smooth steady continuous flow along statistically derived convection patterns, one could anticipate that the gradient drift instability would well describe the evolution of plasma irregularities in patches as they enter and transit the polar cap. However, transient reconnection at the magnetopause has recently been established as a dominant mechanism for patch entry through the cusp and into the polar cap. This is for southward IMF and entry near magnetic noon. For $B_{y}>$ or $<0$, plasma entry is typically along an $\mathrm{L}$ shaped path. The initial entry is at higher speed with a strong ( $B_{y}$ dependent) dawn-dusk velocity component, followed by slower speed convection drift predominantly anti-sunward. The plasma density gradient is taken to have higher density plasma equatorward of the cusp and lower plasma density in the polar cap. The initial injection velocity of the higher density plasma is typically $1-2 \mathrm{~km} / \mathrm{s}$, largely toward the east or west (for respectively $B_{y}<0$ or $B_{y}>0$ ), and driven by the "magnetic tension" force right after reconnection. After the magnetic tension force relaxes, largely anti-sunward flow follows, typically $0.5-1$ $\mathrm{km} / \mathrm{s}$. The starting injection phase initializes irregularities primarily along the sides of the high-density plasma jet via the shear-driven instability. The gradient drift instability operates on the trailing edge, becoming the dominant term in the equation as the patch relaxes into anti-sunward flow to complete the life cycle inside the polar cap.

Key to the case for the new mechanism is the onset time of the observed irregularities. The rise time for plasma structuring upon entering the cusp region is observed to be on the order of a minute or less, as illustrated in Figs. 2 and 3. The rise time for the gradient drift instability acting alone has been calculated to be much longer based on the 2-D theory (Moen et al., 2001, 2002), and slower still for the more recent 3-D theory (Gondarenko and Guzdar, 1999, 2004). In contrast, the theory of Keskinen et al. (1988) leads to a rise time for a shear driven instability of a minute or less for typical cusp conditions, as described and presented herein. For convenience of quantification, we will discuss here the theoretical work by Keskinen and Ossakow (1983) and Keskinen et al. (1988) applied to the scales and conditions given by the data of Carlson et al. (2007), as discussed therein:
1. The growth rate for the GD instability can be written $V /(L(1+\alpha))$ where $V$ is the patch convection velocity, $L$ is the patch density gradient scale length in the direction of convection, and $\alpha=\left(\Omega_{e} \Omega_{i} / v_{e} v_{i}\right)\left(k_{l} / k_{-}\right)^{2}$. Here $\Omega_{e}, \Omega_{i}, v_{e}, v_{i}, k_{l}$, and $k_{-}$are the electron gyrofrequency, ion gyrofrequency, electron collision frequency, ion collision frequency, perturbation wave number parallel to the geomagnetic field, and perturbation wave number perpendicular to the patch convection. For typical polar patch conditions we observed, $\alpha \approx 30$. For $V=1 \mathrm{~km} / \mathrm{s}$ and $L=10 \mathrm{~km}$, the typical GD growth (rise) time is about 5-10 min. However, if the GD is seeded with large amplitude density perturbations from the $\mathrm{KH}$ instability, the GD will appear to grow much more rapidly compared to the growth time from infinitesimal initial perturbations.

2. From Keskinen et al. (1988) we find that the KH growth rate, for ionospheric applications, is a function of a perturbation wave number $k$, the velocity difference $V$, and the velocity difference scale length $L$. A simple estimate from linear theory for the maximized (for wave number $k$ ) $\mathrm{KH}$ growth rate is $0.2 \mathrm{~V} / \mathrm{L}$ (M. J. Keskinen, private communication). The growth or rise time, which is the inverse of the growth rate, is then approximately $5 L / V$. Taking a velocity change of $V=1 \mathrm{~km} / \mathrm{s}$ over a scale distance of $L=10 \mathrm{~km}$, Carlson et al. (2007) got a typical growth (rise) time of $\sim 50 \mathrm{~s}$ for the data reproduced here as Fig. 2. This is in very good agreement with what they proposed as the needed new approach to understand the processes whereby cusp and patch plasma become structured.

In brief, the new cusp plasma structuring process pre-initiates irregularities along the sides (vs. trailing edge) of the patch upon first entry through the cusp. This explains the otherwise unexpectedly early turn on of structure in the plasma right at cusp entry as indicated by other work related to cusp scintillation (Basu et al., 1998), satellite observations (Kivanç and Heelis, 1997, 1998), and collocation of radar backscatter with the cusp (Yeoman et al., 1997; Moen et al., 2001), FTEs (Milan et al., 1999), and onset timing (Moen et al., 2002). This also explains the early downstream patch having structure largely throughout, vs. predominantly just at the trailing edge, as seen on the DMSP satellite (Sa Basu and Su Basu, private communication, 2007). The in-tandem operation of the velocity shear instability followed by the gradient drift instability, in the framework of linear instability theory, should then structure the plasma patches in both the anti-sunward and dawn-dusk directions (examine e.g. the lower panels of Fig. 1).

The well tested GD instability had been known to create irregularities in polar cap patches over their life cycle entering and crossing the polar cap, acting on the trailing edge of the patch. The new finding is the significant acceleration 
of structuring in the cusp, due to the dominance of a different mechanism. The initial injection phase of higher density plasma into the polar cap through the cusp significantly structures the newly entering plasma by the shear driven instability, acting on the sides of the entering patch. Imposition of the initial KH structuring is inherent in the nature of the patch generation mechanism. Its rise time is much faster than that of the GD, which cannot explain the observed rapid onset. It takes the GD too many rise time constants to start from the otherwise weak irregularities that are present (typically $\sim 10$ min vs. $\sim 1 \mathrm{~min})$. However, once the $\mathrm{KH}$ instability has generated strong irregularities, this will let the GD instability start to operate on much larger "pre-initiated" irregularities, and thereby participate more effectively in maintaining them over a smaller number of rise-time-constants. The velocityshear instability leads to initial irregularity formation within $\sim 1$ min after a dense plasma patch enters the cusp region, and subsequently as the plasma patch drifts across the polar cap the gradient-drift instability provides forcing to maintain and lead to further structuring.

\section{Further observations}

We have noted that in the context of this new framework several unexplained aspects and puzzles are explained in in-situ satellite observations and ground-based radio beacon scintillation and radar observations. Much can be learned by reexamining other data within this new framework as well. Velocity shears are a common property of the cusp region, as are associated properties (cusp currents) and consequences (structuring). Below we will highlight some examples.

The work discussed in Figs. 2 and 3 addressed scale sizes of $600-10 \mathrm{~km}$ with the ESR and EISCAT VHF radar and scale sizes of $10 \mathrm{~km}$ to $\sim 100 \mathrm{~m}$ gathered with scintillation receivers. Since we know that larger scale irregularities can seed smaller scale irregularities, Carlson et al. (2007) briefly mentioned that such irregularities could extend down to scale sizes such as those observed by SuperDARN radars, but they did not address it in any further detail. The SuperDARN Hankasalmi radar in Finland is operated by the University of Leicester, and it collected data from the same field-of-view as the ESR, EISCAT VHF, optical instruments, and scintillation receivers. It allows us to further examine this suggestion here.

Figure 2 shows a patch being created in Log $\mathrm{Ne}$ at 07:1507:21 UT (panels a-c and $\mathrm{m}$ ), the onset of flow shear in the ASIP images at 07:18-07:21 UT (panels h-k), and corresponding Ti, Te, and Vi (panels $\mathrm{n}-\mathrm{p}$ ). To facilitate a detailed examination, these parameters ( $\mathrm{Log} \mathrm{Ne}, \mathrm{Vi}, \mathrm{Ti})$ are shown expanded in Fig. 4a-c. Also shown are the MSP data from Ny-Ålesund (Fig. 4d) and high-resolution data from Beam 9 of the SuperDARN Hankasalmi radar (Fig. 4e-g). Because the fields-of-view are not identical, a detailed comparison of radar echoes from one plasma parcel to another must be done
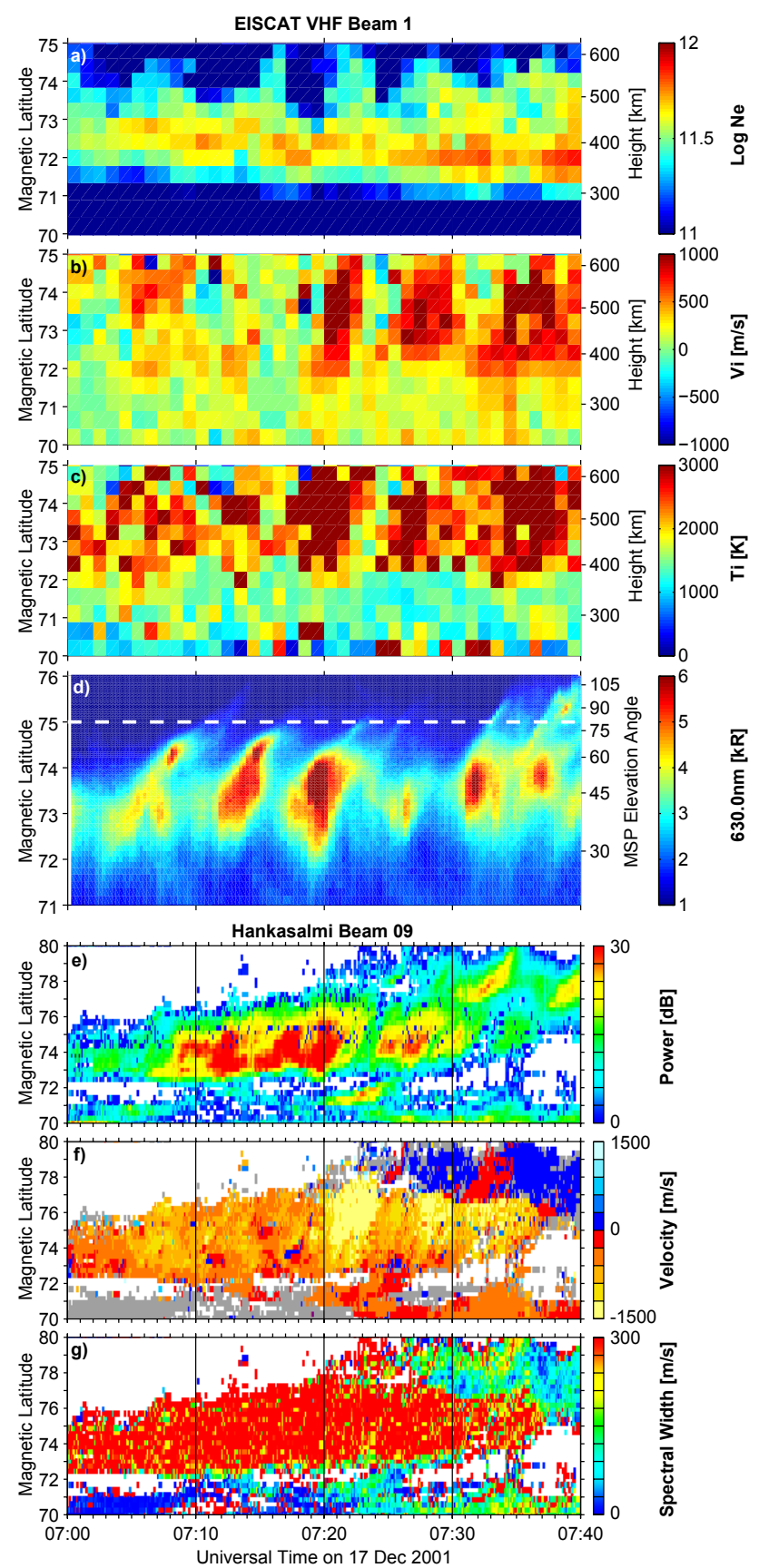

Fig. 4. Panels (a-c) show the parameters Log Ne, Vi and Ti from the EISCAT VHF radar looking over Svalbard for the same time range as in Fig. 2. Panel (d) shows $630.0 \mathrm{~nm}$ emissions from a meridian scanning photometer at Ny-Ålesund. Panels (e-g) show backscatter power, Doppler velocity and spectral width measurements from beam 9 of the SuperDARN Hankasalmi radar in Finland.

with great care. However, velocity shears should by definition be extended in the "east-west" direction, so one could look for signatures in the Hankasalmi data that appear at times and locations similar to the shear signatures in ESR, 

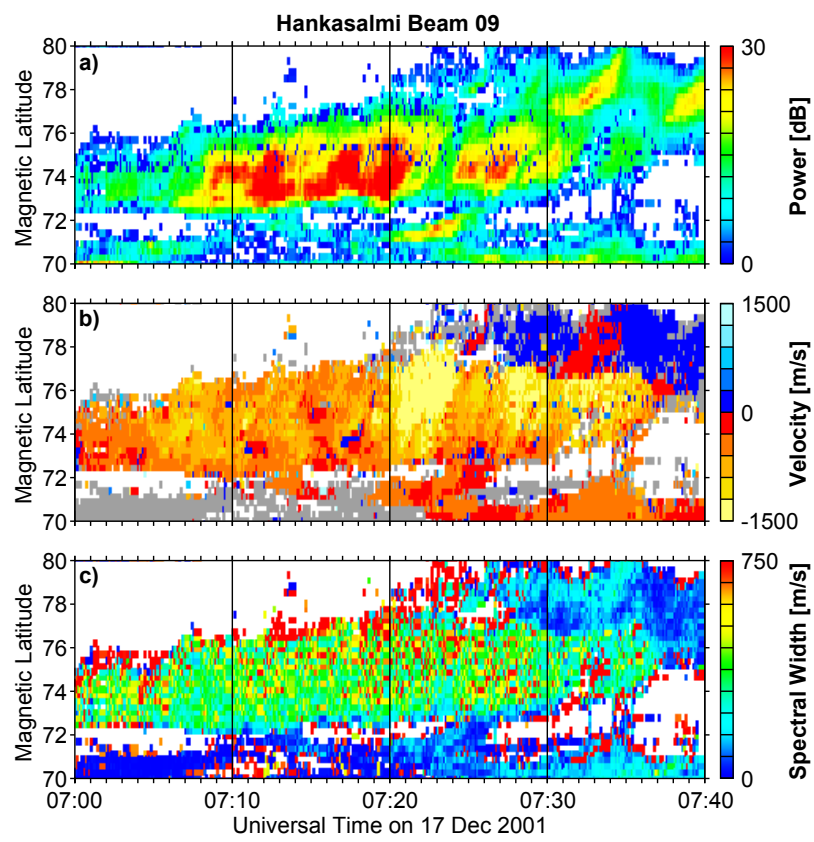

Fig. 5. Same as Fig. 4e-g, except that in panel $\mathrm{c}$ the spectral width scale has been increased from $0-300 \mathrm{~m} / \mathrm{s}$ to $0-750 \mathrm{~m} / \mathrm{s}$ to emphasize structures attributed to large flow shears associated with magnetic reconnection events that trigger the onset of a patch injection from corotation to passage through the cusp with the transpolar cap flow.

EISCAT VHF, and ASIP data. Although shear signatures of the nature we report here have not previously been claimed from SuperDARN data, it is possible that they have not been previously searched for, or not noticed because they could easily be removed by data smoothing or ignored as noise. These flow shears would be small in size, transient in time, and rapidly moving, all making them a challenge to find, and more challenging to believe. With the comprehensive data set at hand, here we can now verify when and where such a signature would be plausible, and we know how rapidly they would move and in what direction.

With all this in mind, let us examine the velocity plot of Fig. 4f during the time span 07:17-07:21 UT and near 72$75^{\circ}$ magnetic latitude. The color scale has been selected so that motion towards the radar is blue, and motion away from the radar is red to yellow. In this time-latitude panel we see a blue shear, about one range gate wide, moving poleward in successive time samples. Without other information it is unlikely that anyone would lay claim to such an obscure feature in itself as being the signature we are searching for. Even here, knowing what to search for, it could be argued to be a chance event, so we need to apply strict criteria to test the statistical significance of these signatures.

The Hankasalmi data in Fig. 4e-g shows episodic events of strongly enhanced backscatter power moving poleward and in locations and with timing consistent with that of the patches in this study, which is neither new nor surprising.
The Hankasalmi Beam 9 is crossing over Longyearbyen and aligned closely towards magnetic north, i.e. the field-of-view is in close proximity to that of the EISCAT radars. The Hankasalmi Beam 9 measurements were obtained every $6 \mathrm{~s}$. Studies have shown that the dayside auroral zone acts as a hard target for HF backscatter radars (Milan et al., 1998), and the broad spectra of $>300 \mathrm{~m} / \mathrm{s}$ in Fig. $4 \mathrm{~g}$ have been determined to be characteristic of the cusp region ionosphere (e.g. Moen et al., 2001, 2002). Figures 2, 3, and 4a-d show five patches initiated by the reconnection events discussed here in the time interval 07:00 to 07:40 UT. In Fig. 4e-g there are several strong backscatter enhancements with a transient nature and a poleward motion in the backscatter power and velocity; a signature that in literature has been linked to PMAFs (Sandholt et al., 2002) in the aurora (e.g. Milan et al., 1999), and to plasma structuring (Milan et al., 2002, 2005), as well as plasma boundaries as identified with complimentary observations (Oksavik et al., 2004a, b, 2005, 2006).

Now let us examine these signatures in closer detail, with a focus on the questions specifically formulated within the context of the new framework. To facilitate the examination we redraw the lower panels of Fig. 4 in Fig. 5, where we have expanded the spectral width scale from $300 \mathrm{~m} / \mathrm{s}$ to $750 \mathrm{~m} / \mathrm{s}$ to allow further discussion of signatures in the spectral width. Figure 6 places the data in a broader context, showing SuperDARN polar cap potential contours and drift vectors. It allows us to determine the large-scale plasma flow over Svalbard (within the field-of-view of the ESR and EISCAT VHF) so that the EISCAT VHF and ESR line-of-sight Vi measurements can be converted to vectors. Figure 6 shows that the flow pattern is very similar from 07:00 until almost 07:30 UT, and the clock angle is also repeatable for these first three frames. IMF $B_{z}$ is about $-7 \mathrm{nT}$, while $B_{y}$ decreases from about -3 to about $-2 \mathrm{nT}$. After 07:30 UT the clock angle becomes more dynamic, the reconnection site appears to move, and the flow pattern starts to change. Since we prefer stable conditions, we choose to focus on the interval 07:00 07:30 UT when the large-scale background flow from SuperDARN is stable (Fig. 6) and EISCAT and scintillation data show repeatable patch formation. Equatorward of $71-72^{\circ}$ magnetic latitude there is no signature of magnetic reconnection with velocity shears and patch formation. Poleward of $73^{\circ}$ there are repeatable patch formation signatures. This is the boundary sector to which we must be attentive, as it is most likely to be rich in flow shears. For starters we note that the commonly used indicator of the cusp in Fig. $4 \mathrm{~g}$ (HF backscatter spectral width $>300 \mathrm{~m} / \mathrm{s}$ ) puts the equatorward boundary of the cusp between $72-73^{\circ}$, consistent with all other data. But what further or new may be found?

Initiating a shear: The first clear signature of increased $S_{4}$ (Fig. 3) appears at 07:04-07:05 UT. In this one minute interval there is one flow shear signature at the same latitude from which the patch is inserted into the poleward flow, a reversal red-to-blue at about $73^{\circ}$ (Fig. 4f), which alone isn't statistically significant. An objective criterion statistically 

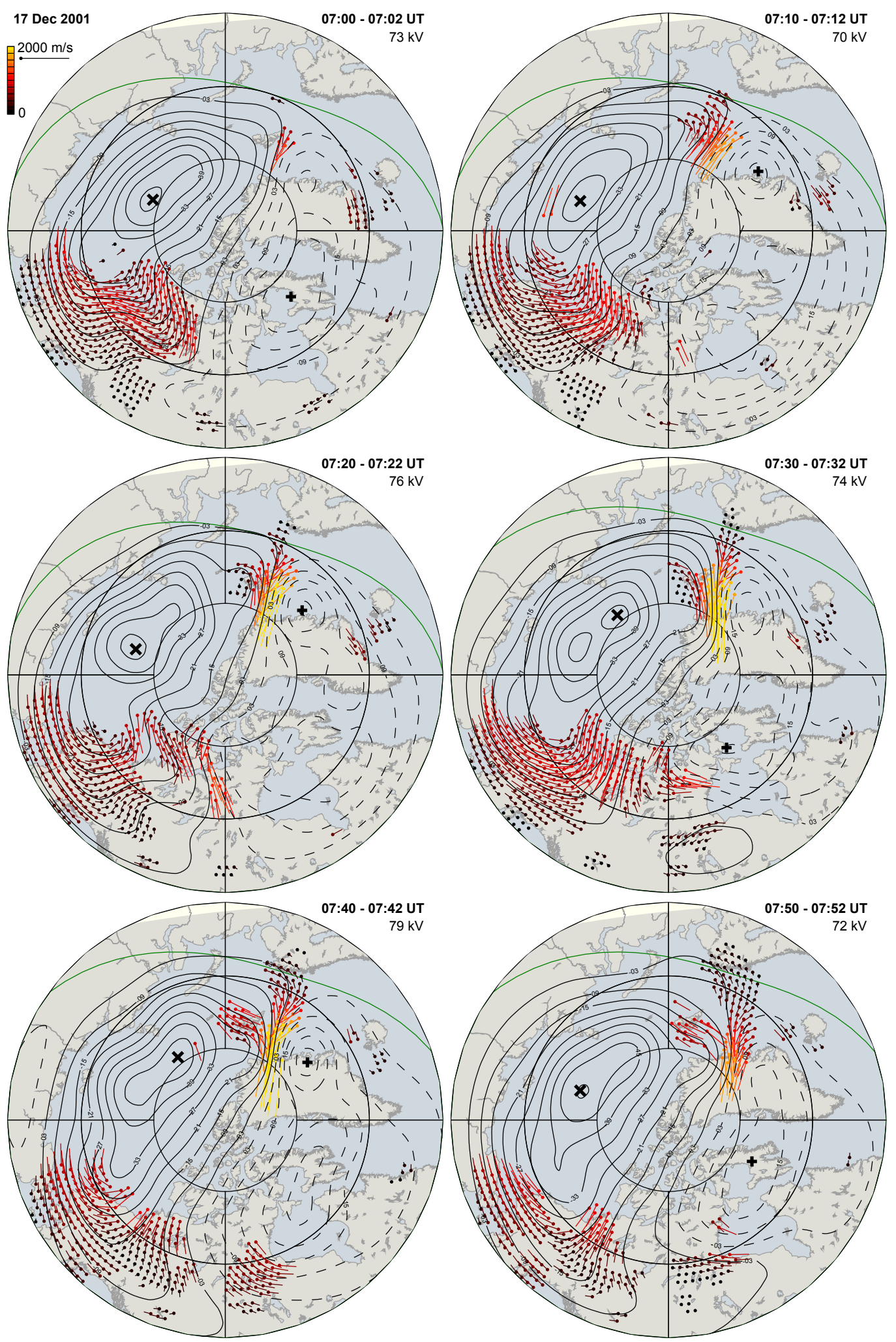

Fig. 6. SuperDARN potential patterns in $10 \mathrm{~min}$ steps during the time range of this study. These data are consistent with a very stable IMF and large-scale flow over Svalbard at 07:00-07:22 UT and a modest change thereafter. 

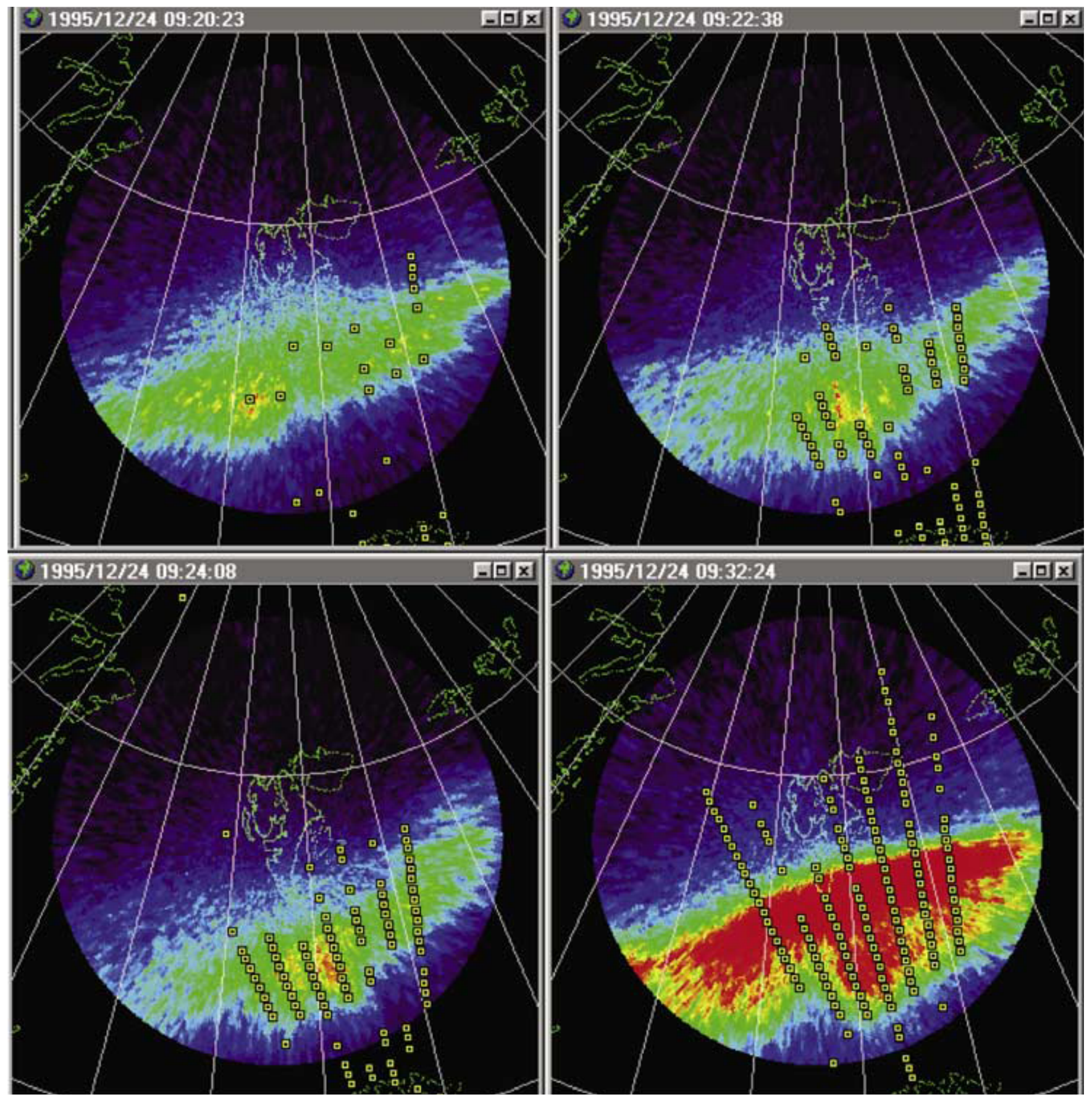

Fig. 7. Overlay of SuperDARN radar echoes on ASIP observations of the cusp over Svalbard. The onset of SuperDARN echoes coincides with the onset time for a brightening of the cusp aurora, interpreted in other studies as a signature of the onset of magnetopause reconnection events. The first three images show a very rapid onset (in two minutes) of SuperDARN echoes from newly formed plasma structures with size similar to the radar wavelength. The fourth frame shows that the initial spatial coincidence, of the equatorward edge of the radar echoes with that of the cusp aurora, still persists $10 \mathrm{~min}$ after onset (adopted from Moen et al., 2001).

significant compared to a random sequence is that a red-bluered pair of reversals must be contiguous in three (or more) successive time samples. The three blue range gates between $72.5^{\circ}$ to $73.5^{\circ}$ at $07: 04-07: 05$ UT in Fig. 4f meet this cri- terion. There are three contiguous pairs of blue-red shear signatures (blue is towards, red is away from the radar) extending over six (or more) data cells. Let us see if there are other contiguous clusters of 6 or more range gates, for which 
at least one or more blue directly abuts at the same range, one or more consecutive reds, including two or more successive range gates increasing in time. A detailed examination shows four events $(07: 04-07: 05,07: 11-07: 12,07: 17-$ 07:18, and 07:25-07:26 UT) that align within a minute of the onset of a patch, and all lie between $72-74^{\circ}$ magnetic latitude. Having identified four such events goes well beyond the reach of random chance.

$H F$ backscatter spectral width: Another question this flow shear framework imposes is to what extent the enhanced spectral width is due to small-scale instability processes in the scattering volume, and to what extent it can be explained by larger-scale shears within a radar range cell. Our examination suggests that both may play a role. For the case of a velocity shear within a single range gate one could expect part of the echo to come from scattering irregularities in the approaching plasma, and part from the receding plasma. It would lead to spectral broadening as long as the Doppler shift is not too excessive (splitting or aliasing the echo). For this reason, in Fig. $5 \mathrm{c}$ we use a $0-750 \mathrm{~m} / \mathrm{s}$ scale to specifically test for spectral width signatures that are appropriate to the velocity shears we find in the complimentary data. Our goal is to check if the spectral width was determined by the velocity shear of the scattering irregularities. The data are consistent with this hypothesis for some (e.g. 07:17-07:20 UT) but not all data in Fig. 5. A visual examination of the $0-750 \mathrm{~m} / \mathrm{s}$ spectral width plots is consistent with signatures for the $3 \mathrm{rd}$ and 4th patch, but the background noise precludes conclusive identification. More objective criteria including statistically significant point-fits to a range of slopes (on a latitude vs. time scale), might extend the number of cases to which this explanation applies. Many spectral broadening mechanisms can apply, and likely do, once a series of patches and more severe plasma instability structures have developed. However, this simple flow shear contribution needs to be included in future explanations.

Area of coverage: The data presented here were collected with emphasis on the propagation of boundaries with time and focusing on a plane near the EISCAT VHF beam and MSP observations of "PMAFs". Figure 7 illustrates that, beyond the 1-D Hankasalmi data presented here, such SuperDARN data can be a tool to map very rapid onsets of cusp irregularities across a large area of the cusp. Moen et al. (2001, 2002) showed a collocation of broad SuperDARN echoes with boundaries in the cusp aurora. Carlson et al. (2007) interpreted those data as a confirmation of the onset of SuperDARN irregularities that were too rapid to be explained without the framework of the new process. Figure 7 illustrates the behavior in 2-D; a collocation with the cusp boundary, a propagation speed of the irregularities across the cusp and into the polar cap, a persistent lifetime of the irregularities as indicated by the echo returns, and an area expected to be affected by scintillation of communication and GPS navigation signals. The spectral width shows a persistent $>300 \mathrm{~m} / \mathrm{s}$ across a large area (vs. apparently large spectral widths at the shear boundary). It could be associated with the turbulence scales of the shear and perhaps GD instability processes as they mature (e.g. Keskinen et al., 1988; Gondarenko and Guzdar, 2004).

Clearly there is far more work to be done with these radars, which is well beyond the scope of this initial illustrative examination. Our goal is just to call attention to a new framework, within which future SuperDARN studies offer much potential. In this paper we have focused on velocity shears that appear in response to magnetic reconnection during periods of southward IMF. However, Rinne et al. (2007) have reported a new class of reverse flow events that produce flow shears in the cusp not only during southward IMF conditions as discussed here, but for both southward and northward IMF. Clearly, much work lies ahead. We hope our new framework will prove useful to further future understanding, and to accelerate future progress for both basis science and practical applications.

Cusp currents and velocity shears: Oksavik et al. (2004a, 2005) called attention to fine-scale velocity shears and finescale currents observed to be characteristic of the cusp (coining the phrase "the cusp current system"). Both the currents and the velocity shears are very likely strongly associated with each other (Moen et al., 2008). A representative case of multiple adjacent shears that is remarkably well documented is shown in Fig. 8. Oksavik et al. (2005) published a very comprehensive dataset (ESR, SuperDARN, DMSP and NOAA satellites) to test for signatures of a flux transfer event (FTE) as predicted by Southwood (1987). The observed and predicted optical and electrodynamics signatures were in excellent agreement for interpreting the plasma flow as an elongated vortex ( $\sim 60 \mathrm{~km}$ wide and $>600 \mathrm{~km}$ long). The test included tracking the radar flow velocity shear (hypothesized Southwood cell) by its optical signature for several hundred $\mathrm{km}$ poleward, where the DMSP satellite crossed the same optical signature and the driftmeter measured in-situ the same flow shear signature as the radar. This shows that the sustained coherent plasma shear was carried in the background convection for over $500 \mathrm{~km}$ and $10 \mathrm{~min}$.

Although the ESR shows a rich spectrum of flow shear scale sizes, it spans only over its resolution limit from $\sim 600 \mathrm{~km}$ to $\sim 10 \mathrm{~km}$. Another complimentary data source is DMSP plasma density and velocity measurements (e.g. Basu et al., 1990) and associated particle fluxes. The DMSP driftmeter can observe scales down to $\sim 1.3 \mathrm{~km}$, but only along the trajectory and not in the required 2-D that is needed to define a velocity shear vs. a gradient. The DMSP pass in Oksavik et al. (2005) showed the presence of structured velocity and electron fluxes (currents) typical of the cusp. Can we go further? For sun aligned arcs the electrodynamics is simple enough that 2-D ASIP images of thin arcs can extend the 1-D satellite velocity gradients into coherent velocity shears (Carlson et al., 1988) as far as an imager can track optical arcs (signatures of current sheets tied to flow shears). The cusp is more complex, but flow gradients of correct sign 

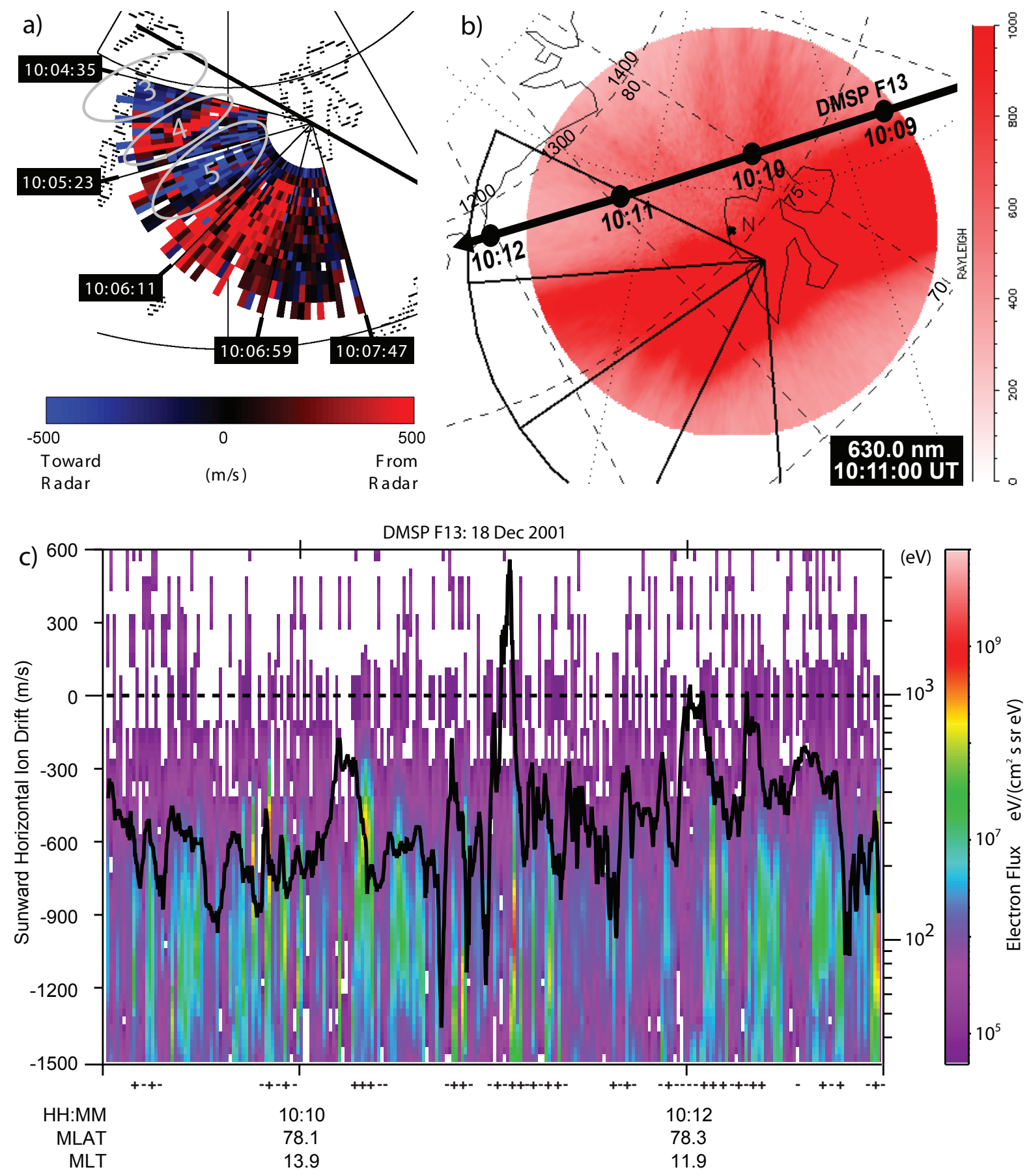

Fig. 8. Panel (a) shows a pair of $>1 \mathrm{~km} / \mathrm{s}$ velocity shears measured within the ESR field-of-view (full red is $500 \mathrm{~m} / \mathrm{s}$ away from the radar, full blue is $500 \mathrm{~m} / \mathrm{s}$ towards the radar, black is $0 \mathrm{~m} / \mathrm{s}$ ). Panel (b) overlays a coincident $630.0 \mathrm{~nm}$ ASIP image on the same area, includes the coincident DMSP F13 satellite track as a thick black line, and shows the ESR field-of-view with thin black lines. The left scale in panel (c) shows the coincident DMSP F13 driftmeter data, and the right scale in panel (c) shows a color spectrogram of the coincident electron particle fluxes $\sim 10^{6}-10^{9} \mathrm{eV} /\left(\mathrm{cm}^{2} \mathrm{~s} \mathrm{sr} \mathrm{eV}\right)$. Near 10:11 UT the driftmeter shows a greater than $\mathrm{km} / \mathrm{s}$ plasma flow shear $(>500 \mathrm{~m} / \mathrm{s}$ sunward to $>500 \mathrm{~m} / \mathrm{s}$ anti-sunward). The sunward plasma flow measured near 10:11 UT by DMSP is the same feature measured by the ESR at 10:05 UT. Its optical signature was tracked poleward for over $10 \mathrm{~min}$ for hundreds of km by the ASIP. These data are distilled from Oksavik et al. (2005). Below panel (c), examples of convergence and divergence regions are indicated “+” and "-_, respectively. 
should align with peaks in the electron flux if the gradient marks a shear (with a collocated current sheet and a possibly detectable optical arc).

Figure $8 \mathrm{a}$ shows a pair of $>1 \mathrm{~km} / \mathrm{s}$ shears measured within the ESR field-of-view (labeled 3-4-5). Figure 8b overlays a coincident atomic oxygen prompt emission $630.0 \mathrm{~nm}$ ASIP image (very similar to a particle excited $777.4 \mathrm{~nm}$ ASIP image not shown) in the same area. The optical filament farthest to the west in Fig. 8b tracked the plasma shear discussed in Fig. 8a. The optical image also shows many other similar poleward drifting filaments. Figure $8 \mathrm{c}$ shows coincident in-situ driftmeter and electron precipitation data from a DMSP pass north of the center of the ASIP image. The green (and yellow) vertical lines are electron flux enhancements seen by DMSP, with the scales on the right hand side. The continuous black line is the in-situ plasma drift component in the Sun-Earth direction, with sunward above the black dashed line and anti-sunward below it. The sole sunward flow spike measured by DMSP near 10:11 UT marks where the satellite crossed the fossil coherent flow shear cell ("FTE flow channel") seen earlier by the ESR at 10:05 UT (Fig. 8a), and tracked with $20 \mathrm{~s}$ resolution by ASIP images. The electron flux current sheet, which was collocated with the observed optical emission, aligns with the expected velocity gradient. The ASIP detects a few other filaments, also found to align with electron flux peaks, on the expected slope of the relative-velocity peak. For simple shear driven arcs the upward current sheet should align with a velocity gradient "down-to-right" (more anti-sunward towards dawn). However, there are more electron flux spikes than what is optically detectable. Some align with the plasma flow gradient associated with current convergence, but not all. Below Fig. 8c is shown where the electron precipitation fluxes coincide or not with local electric field convergence. The +-+- symbols indicate the presence of coincidence, and particle fluxes that appear without these symbols have an absence of coincidence. This is as expected for a mix of current and voltage generator (active and passive arc) conditions. In engineering a constant current generator has internal impedance much larger than its load. On the other hand, a constant voltage generator has internal impedance much smaller than its load. So, ionospheric conductivity variations, from very low to very high values, effectively cause a mix of current and voltage generators.

High-sensitivity ASIPs can be a useful tool to extend flow shear measurements to lower scale sizes; its standalone value bears examination. The flow shear scale size is the natural scale length perpendicular to the flow, over which the flow velocity changes sign. The DMSP driftmeter becomes a powerful tool for highly anisotropic shears as discussed here, since it observes a 1-D cut across velocity shears perpendicular to the satellite track. It is important to study the size of flow shears and plasma density irregularities for future understanding. Likewise the range of scale sizes of the associated fine structure cusp current system needs to be examined within this new context. We see that velocity shear scale sizes clearly extend to at least the km size, so the full range of concern to scintillation is richly filled with structure throughout the cusp.

Energy budget: The transient nature of the flow-spikes and its associated spatial fine structure, vs. the consequences of the slower more continuous flow, has important implications for Poynting flux energy deposition and consequent joule heating of the cusp thermosphere (Carlson, 2007; Luhr et al., 2004; Demars and Schunk, 2007). We will not digress to discuss this in any detail here, but would be remiss to not note its relevance as discussed in these references. Clearly the frictional drag heating associated with plasma motion through the neutral atmosphere is greater if it is an average of transients (as noted in these references) vs. a constant speed case, since the heating depends on the square of the velocity difference between the ions and the neutrals.

Use of the new framework: It is hoped that the new framework, and the rationale leading to it, will motivate further work in many related areas; several of which have been noted here. Theory and modeling must adopt this new framework or process to correctly represent structuring of plasma and patches that enter and transit the polar cap, including related processes in and downstream from the cusp region.

\section{Conclusions}

We have called attention to plasma flow shears as a property characteristic of the cusp near-space environment. We continued to show how this inevitably leads to the need for a replacement of the long accepted GD instability mechanism by the KH instability to first structure the plasma that enters the polar cap through the cusp. We have reviewed how this has led to a new framework for interpreting cusp plasma and polar cap plasma structuring. We describe not a new cusp and polar cap plasma structuring mechanism, but a new process whereby known mechanisms apply and interact to produce new physical consequences. Until 2007 structuring of polar cap patches has been attributed in the literature as due to the gradient drift instability acting alone. Observed onset times of structuring in plasma that enters the cusp are too rapid to be explained by this theory. Plasma patches entering the polar cap through the cusp are in reality subjected to flow shears sufficiently severe as to significantly structure the plasma by the shear driven instability during the initial entry period. This rapidly pre-structures the plasma, after which GD instability processes feed off this rich spectrum of initial structures during the remainder of the transit from the day to the night side polar cap. Theory and modeling must adopt this new framework/process to correctly represent structuring of plasma and patches entering the cusp and transiting the polar cap. We have looked at coincident $\mathrm{Su}-$ perDARN echoes within the context of this new framework, and DMSP data for related work. The two-step process for 
structuring of patches that enter the polar cap gives a simple explanation of why echo returns can turn on abruptly at the edge of the cusp, where reconnection events at the magnetopause lead to strong flow shears accompanying the dominant patch generating mechanism. These strong flow shears in turn very rapidly pre-structure the newly entering patches which then can cause severe scintillation scale structures instantly within the order of a minute, rather than after far longer time and traveled distance into the polar cap. That SuperDARN scale irregularities are found to appear so rapidly, means that cascade to smaller scales is by a similarly rapid composite mechanism. We have illustrated the steps leading to this new framework and further implications, with an especially comprehensive observation set of five successive patches being created and entering the polar cap through the cusp and monitored with ground-based radio, radar, and optical instruments. We called on other data to also bring attention to still further implications of the new framework, including satellite measurements of plasma flow structures and currents, to characterize the fine structured cusp current system and how it could relate to voltage and current generator effects in cusp electrodynamics and energetics.

This new framework must be incorporated into future studies of cusp structuring phenomena for a more realistic understanding. In this paper we have sought to make this point and illustrate some directions of inquiry.

Acknowledgements. EISCAT is an international association supported by the research organizations in China (CRIRP), Finland (SA), France (CNRS, till end 2006), Germany (DFG), Japan (NIPR and STEL), Norway (NFR), Sweden (VR), and the United Kingdom (PPARC). The SuperDARN Hankasalmi radar in Finland is operated by the University of Leicester and supported by PPARC.

Topical Editor K. Kauristie thanks N. Blagoveshchenskaya and S. Milan for their help in evaluating this paper.

\section{References}

Anderson, D. N., Buchau, J., and Heelis, R. A.: Origin of density enhancements in the winter polar cap ionosphere, Radio Sci., 23, 513-519, 1988.

Basu, S., MacKenzie, E., and Basu, Su.: Ionospheric constraints on VHF/UHF communications links during solar maximum and minimum periods, Radio Sci., 23, 363-378, 1988.

Basu, Su., Basu, S., MacKenzie, E., Fougere, P. F., Coley, W. R., Maynard, N. C., Winningham, J. D., Sugiura, M., Hanson, W. B., and Hoegy, W. R.: Simultaneous density and electric field fluctuation spectra associated with velocity shears in the auroral oval, J. Geophys. Res., 93, 115-136, 1988.

Basu, Su., Basu, S., MacKenzie, E., Coley, W. R., Sharber, J. R., and Hoegy, W. R.: Plasma structuring by the gradient drift instability at high latitudes and comparison with velocity shear driven processes, J. Geophys. Res., 95, 7799-7818, 1990.

Basu, S., Weber, E. J., Bullett, T. W., Keskinen, M. J., MacKenzie, E., Doherty, P., Sheehan, R., Kuenzler, H., Ning, P., and Bongiolatti, J.: Characteristics of plasma structuring in the cusp/cleft region at Svalbard, Radio Sci., 33, 1885-1899, 1998.
Basu, Su. and Valladares, C.: Global aspects of plasma structures, J. Atmos. Sol. Terr. Phys., 61, 127-139, 1999.

Carlson Jr., H. C.: The dark polar ionosphere: Progress and future challenges, Radio Sci., 29, 157-165, 1994.

Carlson, H. C.: Role of neutral atmospheric dynamics in cusp density and ionospheric patch formation, Geophys. Res. Lett., 34, L13101, doi:10.1029/2007GL029316, 2007.

Carlson, H. C., Heelis, R. A., Weber, E. J., and Sharber, J. R.: Coherent mesoscale convection patterns during northward interplanetary magnetic field, J. Geophys. Res., 93, 14 501-14 514, 1988.

Carlson, H. C., Oksavik, K., Moen, J., van Eyken, A. P., and Guio, P.: ESR mapping of polar-cap patches in the dark cusp, Geophys. Res. Lett., 29(10), 1386, doi:10.1029/2001GL014087, 2002.

Carlson Jr., H. C., Oksavik, K., Moen, J., and Pedersen, T.: Ionospheric patch formation: Direct measurements of the origin of a polar cap patch, Geophys. Res. Lett., 31, L08806, doi:10.1029/2003GL018166, 2004.

Carlson, H. C., Moen, J., Oksavik, K., Nielsen, C. P., McCrea, I. W., Pedersen, T. R., and Gallop, P.: Direct observations of injection events of subauroral plasma into the polar cap, Geophys. Res. Lett., 33, L05103, doi:10.1029/2005GL025230, 2006.

Carlson, H. C., Pedersen, T., Basu, S., Keskinen, M., and Moen, J.: Case for a new process, not mechanism, for cusp irregularity production, J. Geophys. Res., 112, A11304, doi:10.1029/2007JA012384, 2007.

Cowley, S. W. H. and Lockwood, M.: Excitation and decay of solar wind-driven flows in the magnetosphere-ionosphere system, Ann. Geophys., 10, 103-115, 1992, http://www.ann-geophys.net/10/103/1992/.

Crowley, G.: Critical review of ionospheric patches and blobs, edited by: Stone, W. R., Review of Radio Science 1993-1996, Oxford Sci. Publ., UK, chapter 27, 619-648, 1996.

Demars, H. G. and Schunk, R. W.: Thermospheric response to ion heating in the dayside cusp, J. Atmos. Sol. Terr. Phys., 69, 649660, 2007.

Gondarenko, N. A. and Guzdar, P. N.: Gradient drift instability in high latitude plasma patches: Ion inertial effects, Geophys. Res. Lett., 26, 3345-3348, 1999.

Gondarenko, N. A. and Guzdar, P. N.: Plasma patch structuring by the nonlinear evolution of the gradient drift instability in the high-latitude ionosphere, J. Geophys. Res., 109, A09301, doi:10.1029/2004JA010504, 2004.

Heelis, R. A.: The effects of interplanetary magnetic field orientation on dayside high-latitude ionospheric convection, J. Geophys. Res., 89, 2873-2880, 1984.

Keskinen, M. J. and Ossakow, S. L.: Theories of high-latitude ionospheric irregularities: A review, Radio Sci., 18, 1077-1091, 1983.

Keskinen, M. J., Mitchell, H. G., Fedder, J. A., Satyanarayana, P., Zalesak, S. T., and Huba, J. D.: Nonlinear evolution of the Kelvin-Helmholtz instability in the high-latitude ionosphere, J. Geophys. Res., 93, 137-152, 1988.

Kivanç, Ö. and Heelis, R. A.: Structures in ionospheric number density and velocity associated with polar cap ionization patches, J. Geophys. Res., 102, 307-318, 1997.

Kivanç, Ö. and Heelis, R. A.: Spatial distribution of ionospheric plasma and field structures in the high-latitude F-region, J. Geophys. Res., 103, 6955-6968, 1998. 
Lockwood, M. and Carlson Jr., H. C.: Production of polar cap electron density patches by transient magnetopause reconnection, Geophys. Res. Lett., 19, 1731-1734, 1992.

Lühr, H., Rother, M., Köhler, W., Ritter, P., and Grunwaldt, L.: Thermospheric up-welling in the cusp region: Evidence from CHAMP observations, Geophys. Res. Lett., 31, L06805, doi:10.1029/2003GL019314, 2004.

Milan, S. E., Yeoman, T. K., and Lester, M.: The dayside auroral zone as a hard target for coherent HF radars, Geophys. Res. Lett., 25, 3717-3720, 1998.

Milan, S. E., Lester, M., Cowley, S. W. H., Moen, J., Sandholt, P. E., and Owen, C. J.: Meridian-scanning photometer, coherent HF radar, and magnetometer observations of the cusp: a case study, Ann. Geophys., 17, 159-172, 1999, http://www.ann-geophys.net/17/159/1999/.

Milan, S. E., Lester, M., and Yeoman, T. K.: HF radar polar patch formation revisited: summer and winter variations in dayside plasma structuring, Ann. Geophys., 20, 487-499, 2002, http://www.ann-geophys.net/20/487/2002/.

Milan, S. E., Basu, S., Yeoman, T. K., and Sheehan, R. E.: A comparison of satellite scintillation measurements with HF radar backscatter characteristics, Ann. Geophys., 23, 3451-3455, 2005, http://www.ann-geophys.net/23/3451/2005/.

Moen, J., Carlson, H. C., Milan, S. E., Shumilov, N., Lybekk, B., Sandholt, P. E., and Lester, M.: On the collocation between dayside auroral activity and coherent HF radar backscatter, Ann. Geophys., 18, 1531-1549, 2001, http://www.ann-geophys.net/18/1531/2001/.

Moen, J., Walker, I. K., Kersley, L., and Milan, S. E.: On the generation of cusp HF backscatter irregularities, J. Geophys. Res., 107, 1044, doi:10.1029/2001JA000111, 2002.

Moen, J., Carlson, H. C., Oksavik, K., Nielsen, C. P., Pryse, S. E., Middleton, H. R., McCrea, I. W., and Gallop, P.: EISCAT observations of plasma patches at sub-auroral cusp latitudes, Ann. Geophys., 24, 2363-2374, 2006,

http://www.ann-geophys.net/24/2363/2006/.

Moen, J., Rinne, Y., Carlson, H. C., Oksavik, K., Fujii, R., and Opgenoorth, H.: On the relationship between thin Birkeland current arcs and reversed flow channels in the winter cusp/cleft ionosphere, J. Geophys. Res., 113, A09220, doi:10.1029/2008JA013061, 2008.

Oksavik, K., Moen, J., and Carlson, H. C.: High-resolution observations of the small-scale flow pattern associated with a poleward moving auroral form in the cusp, Geophys. Res. Lett., 31, L11807, doi:10.1029/2004GL019838, 2004a.
Oksavik, K., Søraas, F., Moen, J., Pfaff, R., Davies, J. A., and Lester, M.: Simultaneous optical, CUTLASS HF radar, and FAST spacecraft observations: signatures of boundary layer processes in the cusp, Ann. Geophys., 22, 511-525, 2004b, http://www.ann-geophys.net/22/511/2004/.

Oksavik, K., Moen, J., Carlson, H. C., Greenwald, R. A., Milan, S. E., Lester, M., Denig, W. F., and Barnes, R. J.: Multi-instrument mapping of the small-scale flow dynamics related to a cusp auroral transient, Ann. Geophys., 23, 2657-2670, 2005, http://www.ann-geophys.net/23/2657/2005/.

Oksavik, K., Ruohoniemi, J. M., Greenwald, R. A., Baker, J. B. H., Moen, J., Carlson, H. C., Yeoman, T. K., and Lester, M.: Observations of isolated polar cap patches by the European Incoherent Scatter (EISCAT) Svalbard and Super Dual Auroral Radar Network (SuperDARN) Finland radars, J. Geophys. Res., 111, A05310, doi:10.1029/2005JA011400, 2006.

Rinne, Y., Moen, J., Oksavik, K., and Carlson, H. C.: Reversed flow events in the winter cusp ionosphere observed by the European Incoherent Scatter (EISCAT) Svalbard radar, J. Geophys. Res., 112, A10313, doi:10.1029/2007JA012366, 2007.

Sandholt, P. E., Carlson, H. C., and Egeland, A.: Dayside and polar cap aurora, Astrophysics and Space Science Library, Kluwer Academic Publishers, London, 2002.

Southwood, D. J.: The ionospheric signature of flux transfer events, J. Geophys. Res., 92, 3207-3213, 1987.

Yeoman, T. K., Lester, M., Cowley, S. W. H., Milan, S. E., Moen, J., and Sandholt, P. E.: Simultaneous observations of the cusp in optical, DMSP, and HF radar data, Geophys. Res. Lett., 24, 2251-2254, 1997.

Valladares, C. and Carlson Jr., H. C.: The electrodynamics, thermal, and energetic character of intense sun-aligned arcs in the polar cap, J. Geophys. Res., 96, 1379-1400, 1991.

Valladares, C. E., Decker, D. T., Sheehan, R., and Anderson, D. N.: Modeling the formation of polar cap patches using large plasma flows, Radio Sci., 31, 573-593, 1996.

Valladares, C. E., Alcaydé, D., Rodriguez, J. V., Ruohoniemi, J. M., and van Eyken, A. P.: Observations of plasma density structures in association with the passage of traveling convection vortices and the occurrence of large plasma jets, Ann. Geophys., 17, 1020-1039, 1999, http://www.ann-geophys.net/17/1020/1999/.

Walker, I. K., Moen, J., Kersley, L., and Lorentzen, D. A.: On the possible role of cusp/cleft precipitation in the formation of polarcap patches, Ann. Geophys., 17, 1298-1305, 1999, http://www.ann-geophys.net/17/1298/1999/. 